\title{
Neutrophils mediated multistage nanoparticle delivery for prompting tumor photothermal therapy
}

Bo Ye ${ }^{1 \dagger}$, Bao Zhao ${ }^{2 \dagger}$, Kun Wang ${ }^{3 \dagger}$, Yilong Guo ${ }^{4}$, Qinguo Lu ${ }^{4}$, Longpo Zheng ${ }^{5^{*}}$, Ang Li $6^{{ }^{*}}$ and Jianou Qiao ${ }^{7^{*}}$

\begin{abstract}
Background: Neutrophil-based drug delivery system possesses excellent advantages in targeting at tumour because neutrophils are easily recruited by chemotactic factor in tumor microenvironment. Herein, we developed a novel tactic of multistage neutrophils-based nanoparticle delivery system for promoting photothermal therapy (PTT) of lung cancer.

Results: Au nanorod (AuNR) was successfully modified with bovine serum albumin (AuNRB) and further conjugated with RGD (AuNRBR), followed by neutrophil internalisation to obtain neutrophils-based delivery system (AuNRBR/N). The engineered neutrophils efficiently migrated across the epithelial cells due to inflammatory signal. They exhibited better toxicity against Lewis cells with laser irradiation in vitro. Moreover, AuNRBR/N showed significantly more targetability to tumour tissue compared with cell carrier-free AuNRBR, as demonstrated in Lewis tumour-bearing mice. The enhanced tumour homing efficiency of AuNRBR/N together with subsequently released AuNRBR from the neutrophils was favourable for further deep tissue diffusion and contributed to the inhibition of the tumour growth in PTT and improved survival rate (over 120 days).
\end{abstract}

Conclusions: Overall results illustrated that the design of cell-based nanoparticle delivery system for PTT of cancer is promising.

Keywords: Neutrophils, Photothermal therapy, Inflammatory signal, Au nanorod, Tumour homing

\section{Background}

Photothermal therapy (PTT) is a promising treatment modality for the improvement of cancer therapy, especially for some epidermomas, such as melanoma and breast cancer. Photosensitisers are typically used to

\footnotetext{
*Correspondence: trauma_zlp@163.com; liang@tongji.edu.cn; qjou@163. com

${ }^{\dagger}$ Bo Ye, Bao Zhao, Kun Wang equally contribute to the article

${ }^{5}$ Shanghai Tenth People's Hospital, School of Medicine, Tongji University,

Shanghai 200072, People's Republic of China

${ }^{6}$ School of Life Science and Technology, Tongji University, 1239 Siping

Road, Shanghai 200092, People's Republic of China

${ }^{7}$ Department of Respiratory Medicine, Shanghai Ninth Peoples Hospital,

Shanghai Jiao Tong University School of Medicine, 639 Zhizaoju Rd,

Shanghai 200011, China

Full list of author information is available at the end of the article
}

generate hyperthermia for the elimination of tumour under near-infrared (NIR) irradiation whilst minimising thermal diffusion to normal tissue. Au nanorod (AuNR) with strong NIR absorption was widely studied recently for the presence of plasmonic nanostructures, which serve as highly localised heat sources under NIR irradiation through the photothermal effect [1]. However, systemic administration of AuNR faced many challenges, including poor distribution in vivo and less efficient accumulation within the tumour, which lead to undesired hyperthermia.

Nanoparticles suffer from rapid clearance by the mononuclear phagocytic system (MPS) mostly resident in the liver, thereby effectively impeding the circulation of these nanoparticles in blood and accumulation in

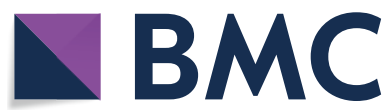

(c) The Author(s) 2020. This article is licensed under a Creative Commons Attribution 4.0 International License, which permits use, sharing, adaptation, distribution and reproduction in any medium or format, as long as you give appropriate credit to the original author(s) and the source, provide a link to the Creative Commons licence, and indicate if changes were made. The images or other third party material in this article are included in the article's Creative Commons licence, unless indicated otherwise in a credit line to the material. If material is not included in the article's Creative Commons licence and your intended use is not permitted by statutory regulation or exceeds the permitted use, you will need to obtain permission directly from the copyright holder. To view a copy of this licence, visit http://creativeco mmons.org/licenses/by/4.0/. The Creative Commons Public Domain Dedication waiver (http://creativecommons.org/publicdomain/ zero/1.0/) applies to the data made available in this article, unless otherwise stated in a credit line to the data. 
tumour [2]. Warren Chan and colleagues reported that only $0.7 \%$ of nanoparticles were successfully delivered to tumour site [3], and this was the main hurdle for the application of AuNR in PTT. The strategy for improving nanoparticle accumulation at tumour has been vigorously pursued. For example, the widely accepted approach to increase in vivo circulation time of AuNR was to modify polyethylene glycols (PEGs) [4]. However, the detection of anti-PEG IgG and IgM indicated accelerated blood clearance of PEG-based AuNR delivery systems from body and hypersensitivity reactions entailing fatal anaphylaxis. Besides, AuNR was modified with targeting molecule (such as folate, RGD, peptide, aptamers and tumour specific antibody) [5-8] to realize the active targeting on tumour. Nevertheless, antibodies and aptamers usually suffer from poor specificity, unfixed configuration and digestion by protease and nucleases. And thus, these strategies did not overcome the limitation of low accumulation in the tumour through its leaky, hastily built vasculature and subsequent poor penetration deeply into the tumour tissue [9].

Cell-based nanoparticle delivery system has achieved great progress in recent years and is another feasible alternative for augmenting tumour targeting. Immune cells, such as monocyte [10], macrophage [11], $\mathrm{T}$ cell [12] and neutrophil [13, 14] were utilised to load or conjugate with therapeutic agents for antitumour therapy. Among these cells, neutrophils can be recruited by chemoattractive agents produced by tumour tissue; which result in the ability of neutrophils to penetrate into the tumour tissue $[13,15]$. When loaded with therapeutic agents, neutrophils act as excellent Trojan horses and could pass across the endothelial barrier and release the cargoes in tumour tissues specifically stimulated by inflammation factors [16]. Neutrophils are used to load liposome-carrying PTX or doxorubicin-loaded magnetic mesoporous silica nanoparticles for the therapy of postoperative malignant glioma [13, 17]. In consequence, neutrophil-based delivery system was a good alternative for improving nanoparticle accumulation and penetration in tumour.

Inspired by this, we constructed a novel cell-based AuNR delivery system by loading AuNR into neutrophils (Fig. 1a, b). The synthetic AuNR was modified with BSA (AuNRB) to promote the biocompatibility that was subsequently internalised by neutrophil. Furthermore, AuNRB was further conjugated with RGD to increase penetration in tumour site after neutrophil delivered AuNRBR, which exhibited good photothermal property, internalisation and toxicity to mouse lung adenocarcinoma Lewis cells in vitro efficiently. In addition, neutrophils carrying AuNRBR could obviously migrate across the epithelial cells due to recruitment by chemotactic factor leading to the promoted targeting efficiency of tumour in vivo. They also exhibit potent inhibition effect on Lewis tumour to enhance the survival rate of mice (Fig. 1c).

\section{Methods \\ Materials}

Hydrochloric acid ( $\mathrm{HCl}, 37 \%)$ was purchased from Sinopharm Chemistry. Foetal bovine serum (FBS), RPMI Media 1640, Dulbecco's modified Eagle's medium (DMEM), penicillin-streptomycin and trypsin were supplied by Gibco Invitrogen. HS-RGD was purchased from GL Biochem (Shanghai), Ltd. FITC-conjugated anti-mouse $\mathrm{CD} 11 \mathrm{~b}$ and $\mathrm{PE} / \mathrm{Cy} 7$-conjugated anti-mouse Ly-6G/Ly-6C were purchase from Tonbo Biosciences. CCK8 and 4,6-diamidino-2-phenylindole (DAPI) were obtained from Beyotime Institute of Biotechnology. Paraformaldehyde (4\%) was obtained from DingGuo Chang Sheng Biotech. Gold (III) chloride trihydrate $\left(\mathrm{HAuCl}_{4} 3 \mathrm{H}_{2} \mathrm{O}\right)$, 4-(N-Maleimidomethyl) cyclohexane1-carboxylic acid 3-sulfo-N-hydroxysuccinimide ester sodium salt (sulfo-smcc) and bovine serum albumin (BSA) was purchased from Sigma-Aldrich (Shanghai) Trading. Phosphate and phosphate buffered saline (PBS, $\mathrm{pH}=7.2,10 \mathrm{mM}$ ) was obtained from Hyclone. Hexadecyl trimethyl ammonium bromide (CTAB), silver nitrate concentrate $\left(\mathrm{AgNO}_{3}\right)$, ascorbic acid, sodium borohydride $\left(\mathrm{NaBH}_{4}\right)$, dimethyl sulfoxide (DMSO), Fluorescein5(6)isothiocyanate (FITC) and formylmethionylleucylphenylalanine (fMLP) were supplied by Aladdin. The BODIPY NHS Ester (succinimidyl ester) and DiD were supplied by Thermo Fisher. Lewis cell line was purchased from American Type Culture Collection (Manassas, VA) and maintained according to American Type Culture Collection-recommended conditions.

\section{Characterisation}

Fluorescence spectra were recorded by a Hitachi F2500 luminescence spectrometer (Hitachi, HongKong). UVVis spectra were characterised by an UV-vis spectrophotometer (Varian, Hong Kong). Transmission electron microscope (TEM, Tecnai-12 Bio-Twin, FEI, Netherlands) was used to observe the nanoparticle morphology. The zeta potential was determined by Zeta Sizer Nano ZS (ZS90, Malvern). Cell viability assay was conducted by using a microplate reader. The cellular uptake was detected using confocal laser scanning microscopy (CLSM, Nikon A1R, Japan). Flow cytometer (FCM, BD FACSVerse) was performed to quantitatively analyse the cellular uptake of nanoparticles. The infrared thermal camera (DALI TECHNOLOGY, LT3-P) was used to record the temperature of the dispersions. The 
inductively coupled plasma-atomic emission spectroscopy (ICP-AES, iCAP 7400) was used to determine $\mathrm{Au}$ concentration.

\section{Synthesis of AuNR, AuNRB and AuNRBR}

AuNR was synthesised by the seeded growth according to the reported the literature with little modification [20]. AuNRB was got by modifying AuNR with BSA based on the method described by Moustafa et al. [21]. Briefly, $1 \mathrm{~mL}$ BSA solution dissolved in the PBS ( $\mathrm{pH}=7.2-7.4$ ) at a concentration of $0.25 \mathrm{mM}$ was added to $10 \mathrm{~mL}$ of the AuNR solution $(0.3 \mathrm{nM})$, and the mixed solution was stirred vigorously for $12 \mathrm{~h}$ at room temperature. Then, the reaction was stopped by removing the excess BSA by centrifugation at $12,000 \mathrm{rpm}$ for $5 \mathrm{~min}$ and the precipitate was resuspended in ultrapure water to gather AuNRB.

AuNRB was suspended in PBS $(2 \mathrm{mg} / \mathrm{mL})$ were respectively functionalised by sulfo-smcc $(150 \mu \mathrm{L}, 5 \mathrm{mg} / \mathrm{mL})$ to endow AuNRB with Mal for further conjugation with HS-RGD. The functionalisation was performed at room temperature (RT) for $1 \mathrm{~h}$. Then, the excessive SulfoSMCC was removed by centrifugation with speed of $10,000 \mathrm{rpm}$ for $10 \mathrm{~min}$ thrice. The purified solution was collected and $100 \mu \mathrm{L}$ HS-RGD $(25 \mathrm{mg} / \mathrm{mL})$ was added by stirring at $350 \mathrm{rpm}$ at RT for $2 \mathrm{~h}$. Afterwards, the reaction was stopped by removing the excess RGD by centrifugation with speed of 10,000 rpm for $10 \mathrm{~min}$ for 3 times and the solution was resuspended in ultrapure water with final volume of $2 \mathrm{~mL}$. The sample was stored at $4{ }^{\circ} \mathrm{C}$ for further study.

FITC DMSO solution was reacted with BSA at a molar ratio of 3: 1 (FITC: BSA) for $6 \mathrm{~h}$. After the ultrafiltration with water to remove free FITC, FITC labelled BSA was collected and used to synthsize FITC-AuNRBR and FITC-AuNRB.

\section{In vitro photothermal effect}

AuNRBR dispersed in PBS with the concentration of 10, 20,40 and $80 \mu \mathrm{g} / \mathrm{mL}$ in a tube was irradiated with an $808 \mathrm{~nm}$ laser at the power density of $1 \mathrm{~W} / \mathrm{cm}^{2}$ for $8 \mathrm{~min}$. The temperature increase was recorded at $1,2,3,4,5,6$, 7 and $8 \mathrm{~min}$, and the thermal images of the dispersions were recorded at $8 \mathrm{~min}$ by an infrared thermal camera, respectively. PBS was used as control treated under the same conditions.

\section{Isolation and purity of neutrophils from mice}

Neutrophils were isolated from femurs and tibias of 3-week-old to 4-week-old C57BL/6 mice. Cells isolated from femurs and tibias were suspended in PBS and centrifuged at $1000 \mathrm{rpm}$ for $3 \mathrm{~min}$. The collected cells were resuspended with $2 \mathrm{~mL}$ separation mixed solution containing 55\%, 65\% and 78\% (v:v) Percoll in PBS, followed by centrifugation for $30 \mathrm{~min}$ at $500 \mathrm{~g}$. Afterwards,

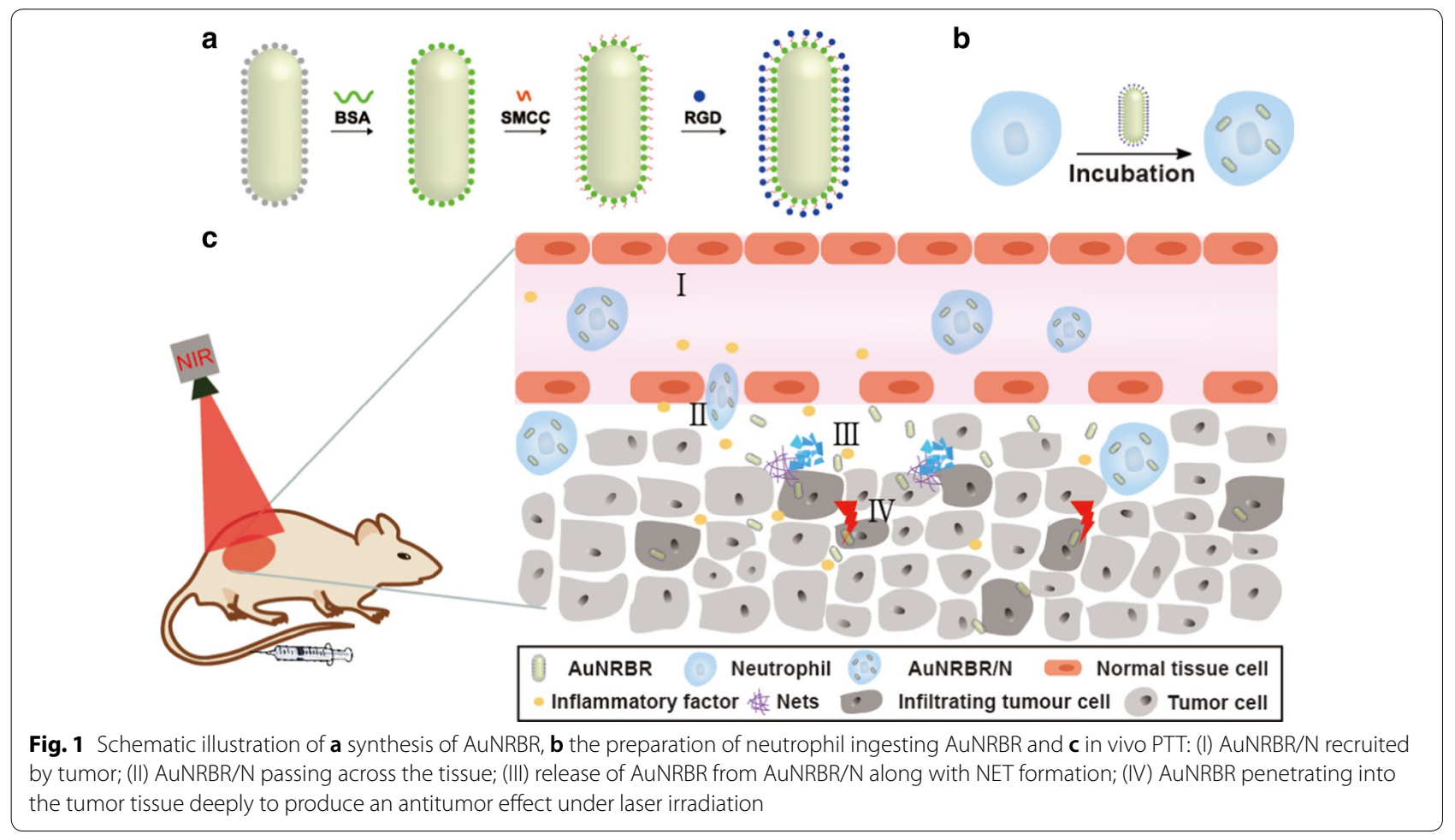


neutrophils were collected at the interface of the $65 \%$ and $78 \%$ fractions and further washed by ice-cold PBS thrice.

To identify the purity of neutrophils, they were doubly stained with FITC-conjugated anti-mouse CD11b and $\mathrm{PE} / \mathrm{Cy} 7$-conjugated anti-mouse Ly-6G/Ly-6C to analyse by flow cytometry. Neutrophils were also directly observed by microscope.

\section{AuNRBR loaded into neutrophils (AuNRBR/N)}

AuNRBR was incubated with neutrophils to produce AuNRBR/N. To measure the loading efficiency, AuNRBR was labelled with FITC $\left(1 \mu \mathrm{g} / 10^{5}\right.$ cells $)$ and incubated with neutrophils for 10,30 and $60 \mathrm{~min}$ at $37{ }^{\circ} \mathrm{C}$ with slight shaking. Afterwards, the cells were centrifuged at $1200 \mathrm{rpm}$ for $5 \mathrm{~min}$ to remove excess AuNRBR-FITC. Finally, the cells were analysed by flow cytometry and observed by fluorescence microscope.

\section{AuNRBR release from AuNRBR/N}

The release behaviour of AuNRBR from AuNRBR/N was investigated by determining the amount of AuNRBR in supernatant. AuNRBR/N was resuspended in RPMI 1640, followed by irradiation with $808 \mathrm{~nm}$ laser. Then, the cell suspension was cultured with $5 \% \mathrm{CO}_{2}$ at $37^{\circ} \mathrm{C}$. At the interval time point $(0,1,2,4,8$ and $12 \mathrm{~h})$, the cell suspension was centrifuged at $2000 \mathrm{~g}$ for $5 \mathrm{~min}$. The supernatant was collected, and the AuNRBR content was determined by ICP-AES. Additionally, AuNRBR/N suspension with no laser irradiation was treated with the same process used in the control.

\section{Cellular uptake of AuNRB and AuNRBR}

Lewis cells were planted in 6-well plate with DMEM containing $10 \% \mathrm{FBS}$ and incubated at $37{ }^{\circ} \mathrm{C}$ and $5 \%$ $\mathrm{CO}_{2}$. They were allowed to grow until about $70 \%$ confluency. Then, the medium was removed and replaced with $2.5 \mathrm{~mL}$ serum-free DMEM with FITC labelled AuNRB and AuNRBR (equivalent FITC, quantified by fluorescence spectra) and incubated for $4 \mathrm{~h}$, respectively. After incubation, Lewis cells were washed twice with PBS and collected for uptake analysis by flow cytometry. To more directly observe the uptake of FITC labelled AuNRB and AuNRBR by Lewis cells, the same operation as above was performed except for the planting of Lewis cells in laser confocal dishes. The cells washed with PBS were fixed with $4 \%$ paraformaldehyde and counterstained with DAPI for further observation by confocal laser scanning microscopy (CLSM).

\section{Endothelial permeability}

The permeability was determined by using a transwell system (5 $\mu \mathrm{m}$ pore size, EMD Millipore Corporation). Briefly, human umbilical vein endothelial cells (HUVECs) were seeded in the upper chamber of the transwell in the 24-well plate and cultured with complete medium. The tightness of the cell monolayer was determined by measuring the trans-endothelial electrical resistance (TEER) value. When the TEER value arrive at or beyond $200 \Omega \mathrm{cm}^{2}$, the cell monolayer was used for the transmigration studies.

AuNRBR/N was added into upper chamber to coincubate with HUVECs for $4 \mathrm{~h}$, as well as serum-free medium added into the lower chamber in the presence or absence of fMLP. Then, the media in the upper and lower chambers were collected, and the cell monolayer on the transwell membrane was harvested. The AuNRBR in the supernatant, intracellular and filtrate compartments was measured by ICP-AES to further calculate the ratio by comparing with the input amount of AuNRBR.

\section{Cell viability}

Lewis cells were planted in 96-well plate with DMEM containing 10\% FBS and incubated for $24 \mathrm{~h}$. Afterwards, the medium was removed and replaced with $100 \mu \mathrm{L}$ serum-free DMEM containing AuNRBR/N (Au concentration of 10, 20,40,60,80 $\mu \mathrm{g} / \mathrm{mL}$ ) and incubated for 6 and $12 \mathrm{~h}$, respectively. Then, the medium was removed and washed with PBS twice. Another $200 \mu \mathrm{L}$ of DMEM was added prior to irradiation with or without an $808 \mathrm{~nm}$ laser for $5 \mathrm{~min}\left(0.5 \mathrm{~W} / \mathrm{cm}^{2}\right)$. Following incubation overnight at $37{ }^{\circ} \mathrm{C}$, the cell viability was determined by the above mentioned CCK- 8 protocol.

\section{In vivo tumour targeting}

The mice (C57BL/6, 5-6 weeks, female) were injected subcutaneously with Lewis cells to build tumour-bearing mice model and divide into 3 groups (3 mice per group). When the tumour volume reached about $200 \mathrm{~mm}^{3}$, the fluorescence dye (BODIPY)-labelled AuNRBR and AuNRBR/N (equivalent fluorescence dye) were injected intravenously to the mice. At 4, 8, 12, 24 and 48 h postinjection, the images were taken. For further analysis of targeting efficiency, the major organs and tumours were taken out to image at $12 \mathrm{~h}$ post-injection. Additionally, the tumour tissues were fixed with paraformaldehyde (4\%) and cut into $20 \mu \mathrm{m}$ sections, untreated tumourbearing mice tissue used as negative control. The slides were observed by CLSM only after staining with DAPI. 
To further analyse the ratio of the administered neutrophils to total neutrophils in tumour, the AuNRBR/N stained with $\mathrm{DiD}$ was injected intravenously into tumour-bearing mice. Then, the mice were sacrificed at each individual time point $(4,8,12,24$ and $48 \mathrm{~h})$, and the tumour tissues were collected. Subsequently, the tumour tissues were digested to make a cell suspension, followed by staining with PE/Cy7-conjugated anti-mouse Ly-6G/ Ly-6C and analysis by flow cytometry.

\section{In vivo photothermal effect}

AuNRBR/N and AuNRBR dispersed in PBS at a concentration of $100 \mu \mathrm{g} / \mathrm{mL}$ were injected intravenously into tumour-bearing mice. After $12 \mathrm{~h}$, the mice were irradiated with an $808 \mathrm{~nm}$ laser at the power density of $0.5 \mathrm{~W} /$ $\mathrm{cm}^{2}$ for $5 \mathrm{~min}$. The temperature increase was recorded at $0,1,3$ and $5 \mathrm{~min}$, and the thermal images of the AuNRBR/N-treated mice were recorded. PBS was used as control and treated under the same conditions.

\section{In vivo antitumour therapy}

The tumour-bearing mice models were built as described above. Twenty tumour-bearing mice were randomly divided into four groups with five mice per group and intravenously injected with AuNRBR/N, AuNRBR and AuNR with equivalent doses of Au $(100 \mu \mathrm{g})$ every 3 days for 3 times per day. The mice injected with PBS were used as the control. The tumours of mice were exposed to $808 \mathrm{~nm}$ laser irradiation $\left(0.5 \mathrm{~W} / \mathrm{cm}^{2}\right)$ for $5 \mathrm{~min}$ at $12 \mathrm{~h}$ post-injection. The tumour size was measured, and body weight was recorded every other day along with the day the mice died. The tumour volume was calculated through the formula: $\mathrm{V}=\left(\mathrm{L} \times \mathrm{W}^{2}\right) / 2$ (L, tumour length; $\mathrm{W}$, tumour width). On day 15 , the mice were sacrificed, and tumours were extracted for imaging and further fixed in $4 \%$ paraformaldehyde for H\&E assay according to the protocol.

\section{Statistical analysis}

The statistical significance of the differences between sphere groups was calculated using a one-way ANOVA test with SPSS 19.0 (SPSS Inc., USA). Significant differences between or among the groups are implied by * $\mathrm{p}<0.05,{ }^{* *} \mathrm{p}<0.01,{ }^{* * * *} \mathrm{p}<0.001$.

\section{Results and discussion}

\section{Synthesis and characterisations of AuNR, AuNRB} and AuNRBR

AuNR was successfully synthesised with an average size of around $50 \mathrm{~nm}$, as observed by TEM (Fig. 2a). The zeta potential of AuNR (Fig. 2c) was around $+35 \mathrm{mV}$, which was due to the cationic surfactant of CTAB. The AuNR was modified with BSA to increase biocompatibility, as demonstrated by the TEM (Fig. 2b). As shown in Fig. 2c, the zeta potential decreased to around $-18 \mathrm{mV}$ compared with AuNR, thereby suggesting the successful conjugation of BSA to the surface of AuNR. To further identify the successful modification of BSA, UV was used to determine that the transverse and longitudinal peaks of AuNRB experienced redshift compared with AuNR (Fig. 2d), which was due to the plasmonic coupling of AuNR in this system. Moreover, AuNRB was conjugated with RGD by characterisation with the zeta potential of around $-17 \mathrm{mV}$ (Fig. 2c). Besides, RGD labelled with BODIPY was conjugated to AuNRB along with the change of the solution colour from pink to blue after purification (Fig. 2c, insertion), thereby indicating that RGD was successfully conjugated to AuNRB. The photothermal property of AuNRBR was studied by using an $808 \mathrm{~nm}$ laser. Figure 2e shows that the temperature of the solutions rose was dependent on the concentration of AuNRBR. Interestingly, AuNRBR solutions $\left(C_{\mathrm{Au}}=80 \mu \mathrm{g} /\right.$ $\mathrm{mL}$ ) exhibited rather high temperature, which increased up to $50{ }^{\circ} \mathrm{C}$ within $5 \mathrm{~min}$. In addition, the thermal images of AuNRBR had good photothermal conversion efficiency, thereby indicating the good photothermal property of AuNRBR due to the plasmonic coupling effect (Fig. 2f).

\section{Neutrophils loading AuNRBR and the release efficiency when irradiated with an $808 \mathrm{~nm}$ laser}

Firstly, the neutrophils were isolated from the mice bone marrow. The purified neutrophils were doubly stained with FITC-CD11b and PE/Cy7 Ly-6G/Ly-6C and analysed by flow cytometry. The yield was about $2.5 \times 10^{6}$ cells/mouse, and the purity of neutrophils was approximately 95\% (Fig. 3a, b). The morphology of the obtained neutrophils was observed directly under the microscope. They were polymorphonuclear and small, with sizes of approximately $8 \mu \mathrm{m}$ (Fig. 3c).

Considering the short lifespan (only a few hours after isolation from blood) and quick phagocytosis ability of neutrophils, the incubation time was optimized to ensure the effective loading amount of AuNRBR. Figure 3d shows that neutrophils incubated with FITC-labelled AuNRBR showed more fluorescence signals with time going by according to flow cytometry analysis results. At the post-incubation time of $60 \mathrm{~min}$, the fluorescence intensity was the highest, thereby suggesting that the ideal incubation time in which viability was not affected was $60 \mathrm{~min}$. The neutrophils loading AuNRBR were also observed by CLSM. The same conclusion was drawn, i.e. neutrophils incubated with AuNRBR for 60 min showed stronger green fluorescence compared with those incubated for $10 \mathrm{~min}$ or $30 \mathrm{~min}$, as observed by CLSM 
(Fig. 3e). Therefore, the incubation time of 60 min was particularly well suited to load AuNRBR into neutrophils.

To investigate AuNRBR release mediated by $808 \mathrm{~nm}$ laser irradiation, the content of AuNRBR was determined at each interval time. As shown in Fig. 3f, most AuNRBR remained in neutrophils, and only around 15\% AuNRBR was detected in the medium at $12 \mathrm{~h}$, thereby indicating that AuNRBR/N was stable in a normal physiological environment. However, a rather rapid release of AuNRBR from AuNRBR/N occurred when irradiated with an $808 \mathrm{~nm}$ laser. Approximately 80\% AuNRBR was released from AuNRBR/N within $2 \mathrm{~h}$. AuNRBR was capable of being released efficiently from AuNRBR/N under laser irradiation, thereby further exerting antitumour function.

\section{Cellular uptake and cytotoxicity of AuNRBR and AuNRBR/N}

The cell uptake was estimated by using CLSM and flow cytometry to prove the targeting effect of AuNRBR. As shown in Fig. 4a, Lewis cells incubated with AuNRBR showed stronger fluorescence signals compared with AuNRB, as observed by CLSM. The cellular uptake was further measured quantitatively by flow cytometry. As shown in Fig. 4b, the mean fluorescence intensity of AuNRBR was much higher than that of AuNRB, which implied that the modification of RGD could increase the cellular uptake efficiently. The phenomenon above was due to the specific binding of RGD to integrin receptors expressed on Lewis cells [18].

Next, we detected the cytotoxicity of AuNRBR and AuNRBR/N to tumor cells with or without laser irradiation. As illustrated in Fig. 4c, AuNRBR/N exhibited little cytotoxicity to Lewis cells at 6 and $12 \mathrm{~h}$ post-incubation without laser irradiation, which demonstrated the excellent biosafety of AuNRBR/N without laser irradiation and selectivity for tumour ablation in PTT. In contrast, AuNRBR/N was able to inhibit the proliferation of Lewis cells effectively both at 6 and $12 \mathrm{~h}$ post-incubation with laser irradiation (Fig. 4d). Higher concentration of $\mathrm{Au}$ (40, 60 and $80 \mu \mathrm{g} / \mathrm{mL}$ ) displayed stronger toxicity, in which Lewis cell viability was below $50 \%$ and close to around $10 \%$. The potent cytotoxicity of AuNRBR/N was ascribed to the stronger photothermal effect of AuNRBR and extracellular trap net released from neutrophils [19]. Moreover, the cell viability of group AuNRBR/N at $12 \mathrm{~h}$ post-incubation decreased compared with that at $6 \mathrm{~h}$ post-incubation, indicating that more AuNRBR was delivered to Lewis cells with extended incubation time. Therefore, AuNRBR/N was capable of killing tumour cell in vitro with laser irradiation. a
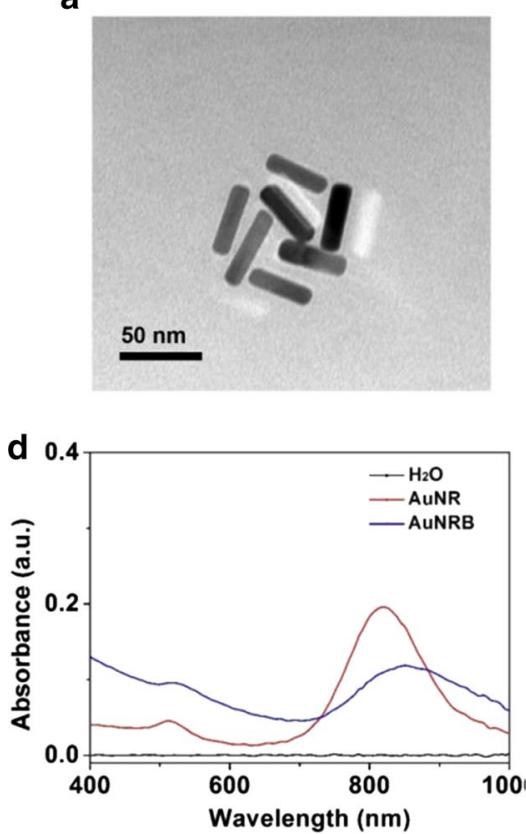

b
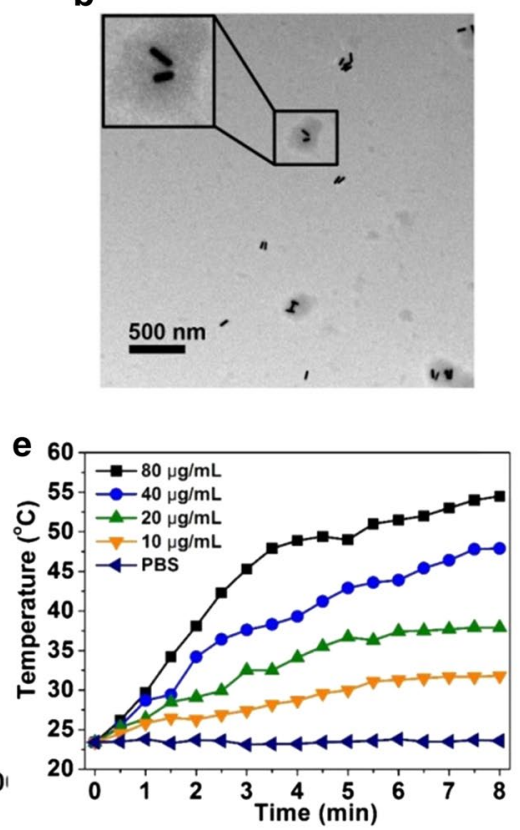

C

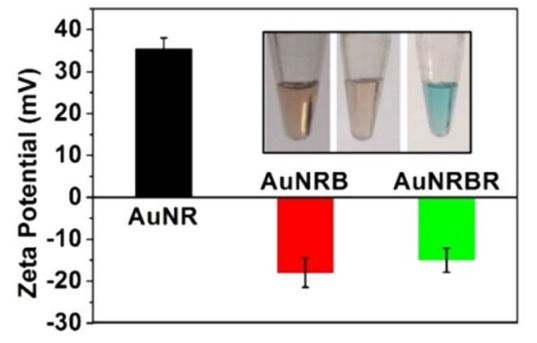

$\mathbf{f}$

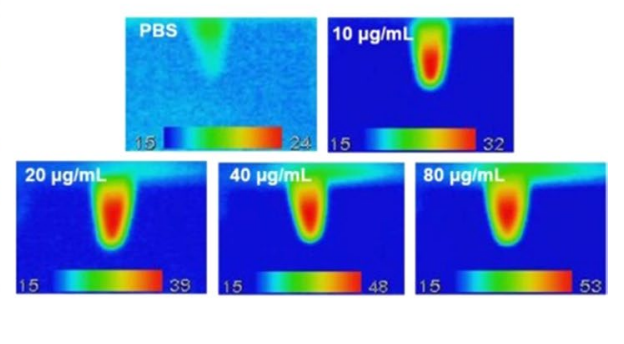

Fig. 2 TEM of a AuNR and $\mathbf{b}$ AuNRB; $\mathbf{c}$ zeta potential of AuNR, AuNRB and AuNRBR, insertion of the images of the solution of AuNR, AuNRB and BODIPY labeled RGD conjugated with AuNRB. $\mathbf{d}$ UV measurement of AuNR and AuNRB. e Photothermal property of AuNRBR with different concentrations gradient. $\mathbf{f}$ In vitro infrared thermal images of various concentrations $(0,10,20,40$ and $80 \mu \mathrm{g} / \mathrm{mL})$ immediately after illumination. The error bars were presented as mean $\pm S D(n=3)$ 


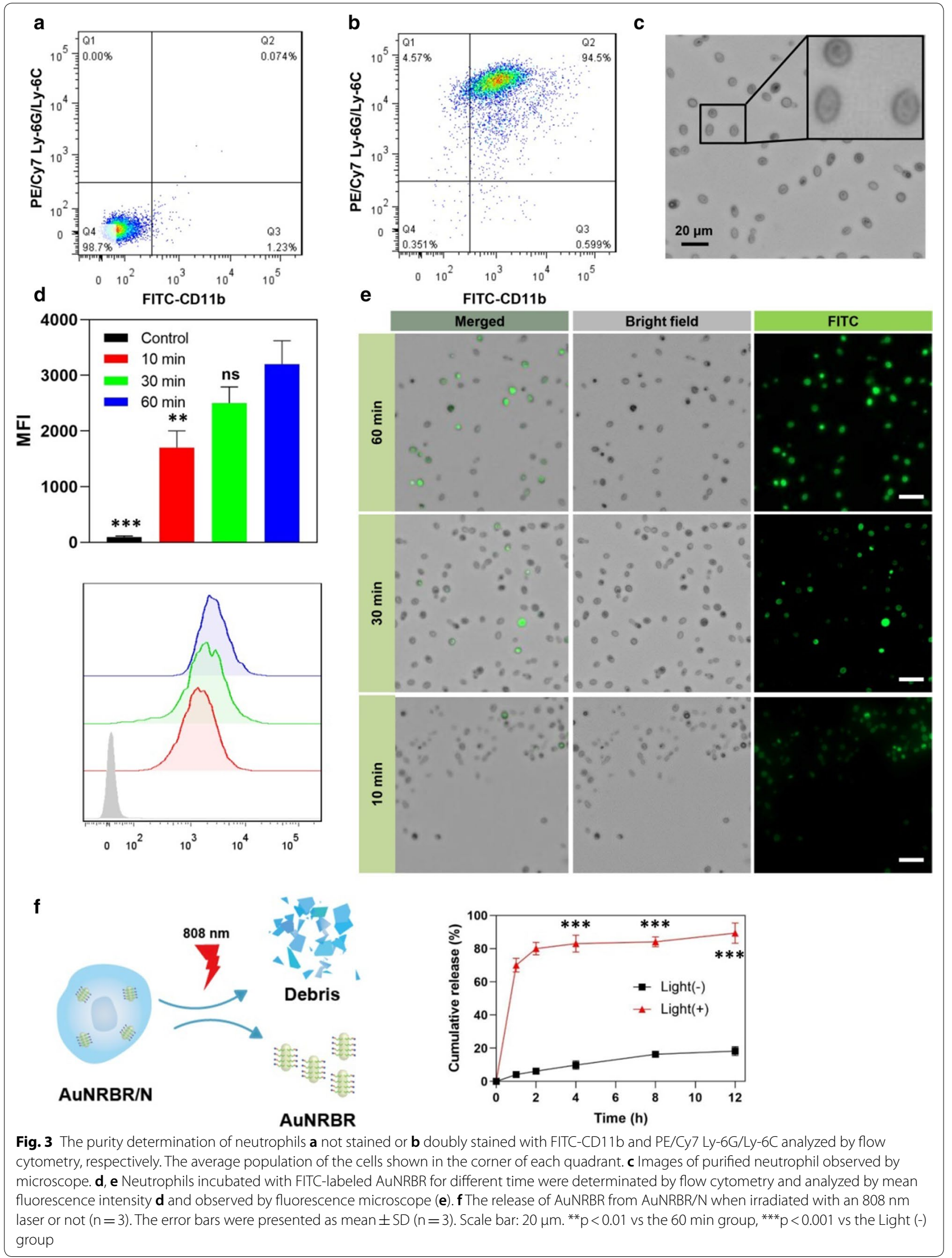




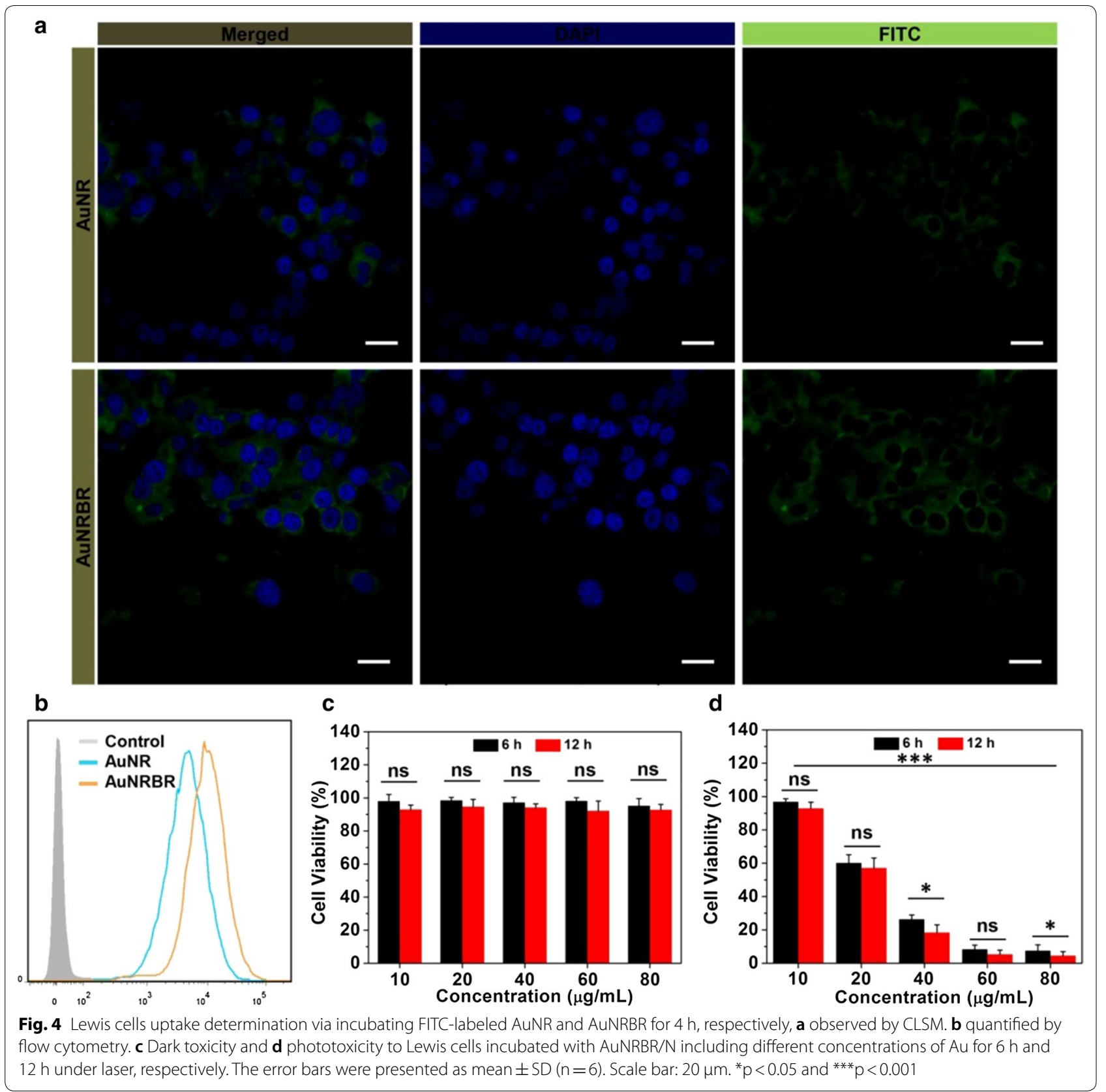

\section{AuNRBR/N promote endothelial permeability in vitro and tumour targeting in vivo}

The microvascular endothelial cell penetrating ability of AuNRBR/N was studied by using a transwell technology (Fig. 5a) and by using the HUVEC as the cell monolayer. As shown in Fig. 5b, AuNRBR/N exhibited lower permeability (2\%) through the monolayer in the absence of fMLP, and nearly all AuNRBR/N stayed in the upper chamber (86\%), thereby suggesting that AuNRBR/N failed to pass through the endothelium layer efficiently. However, the amount of AuNRBR increased up to about
$38 \%$ in the presence of fMLP, along with a decreased percentage of AuNRBR in the upper chamber (50\%). The results suggested that AuNRBR/N could be recruited by inflammatory factor and then migrate across blood vessels into the tumour site efficiently.

Next, to evaluate the elevated tumour targeting efficiency and endothelial permeability of AuNRBR/N and AuNRBR in vivo, AuNRBR/N and AuNRBR were intravenously injected into Lewis tumour-bearing mice and real-time fluorescence imaging technique to detect the fluorescence intensity in tumor. Compared with 
a

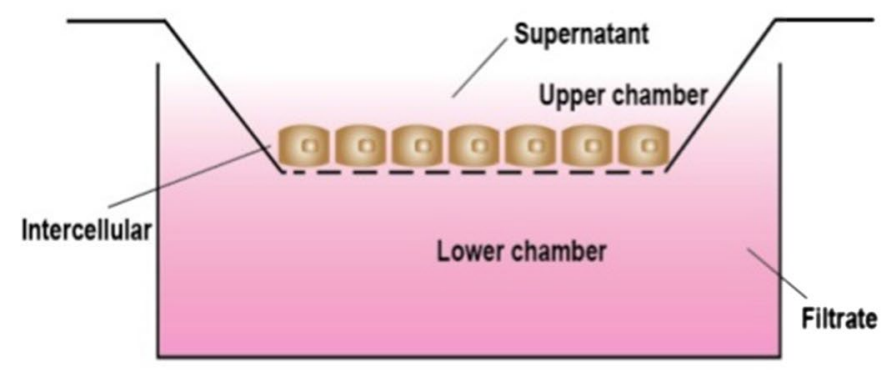

C

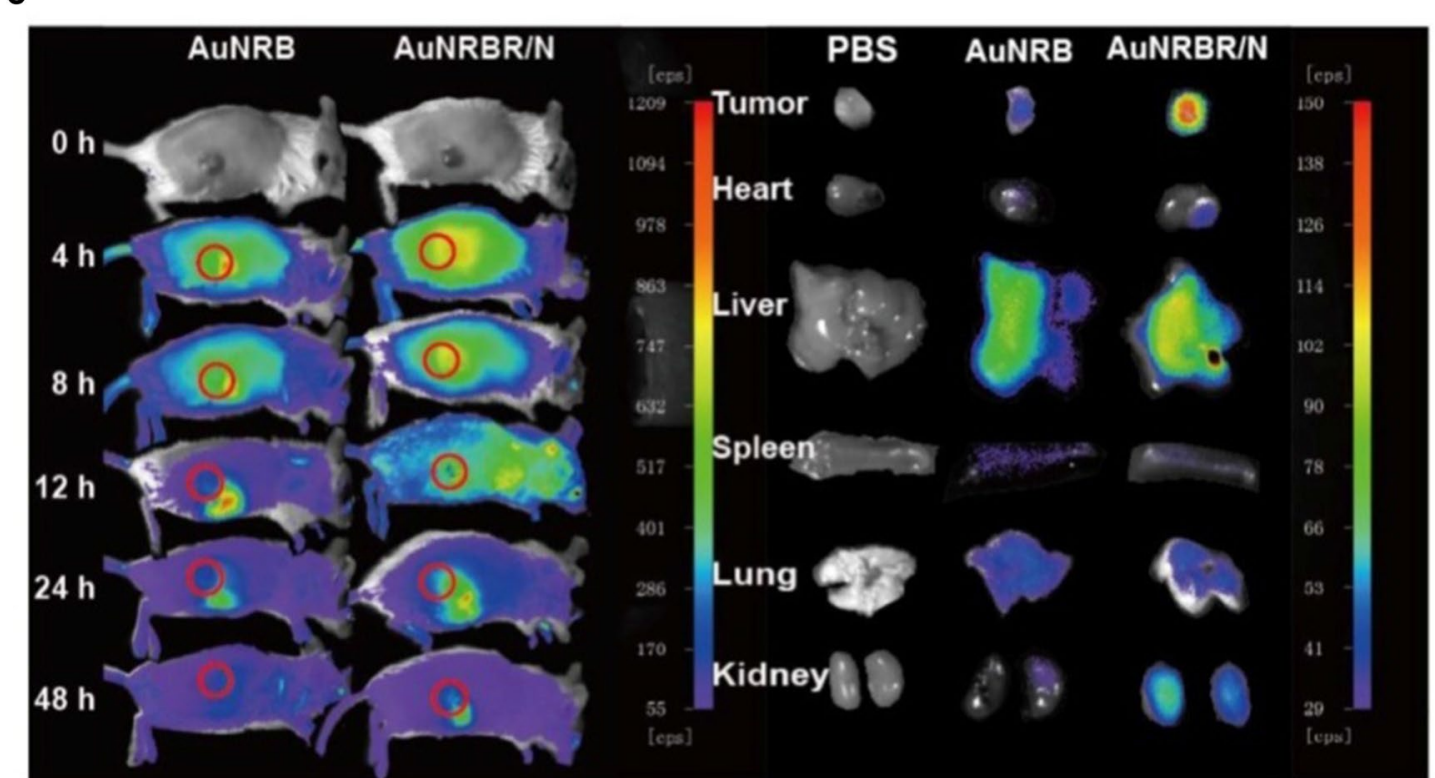

\section{b}

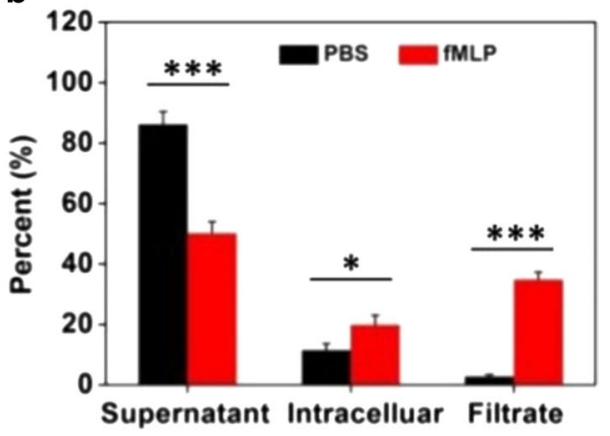


Ye et al. J Nanobiotechnol

(2020) 18:138

Page 10 of 14

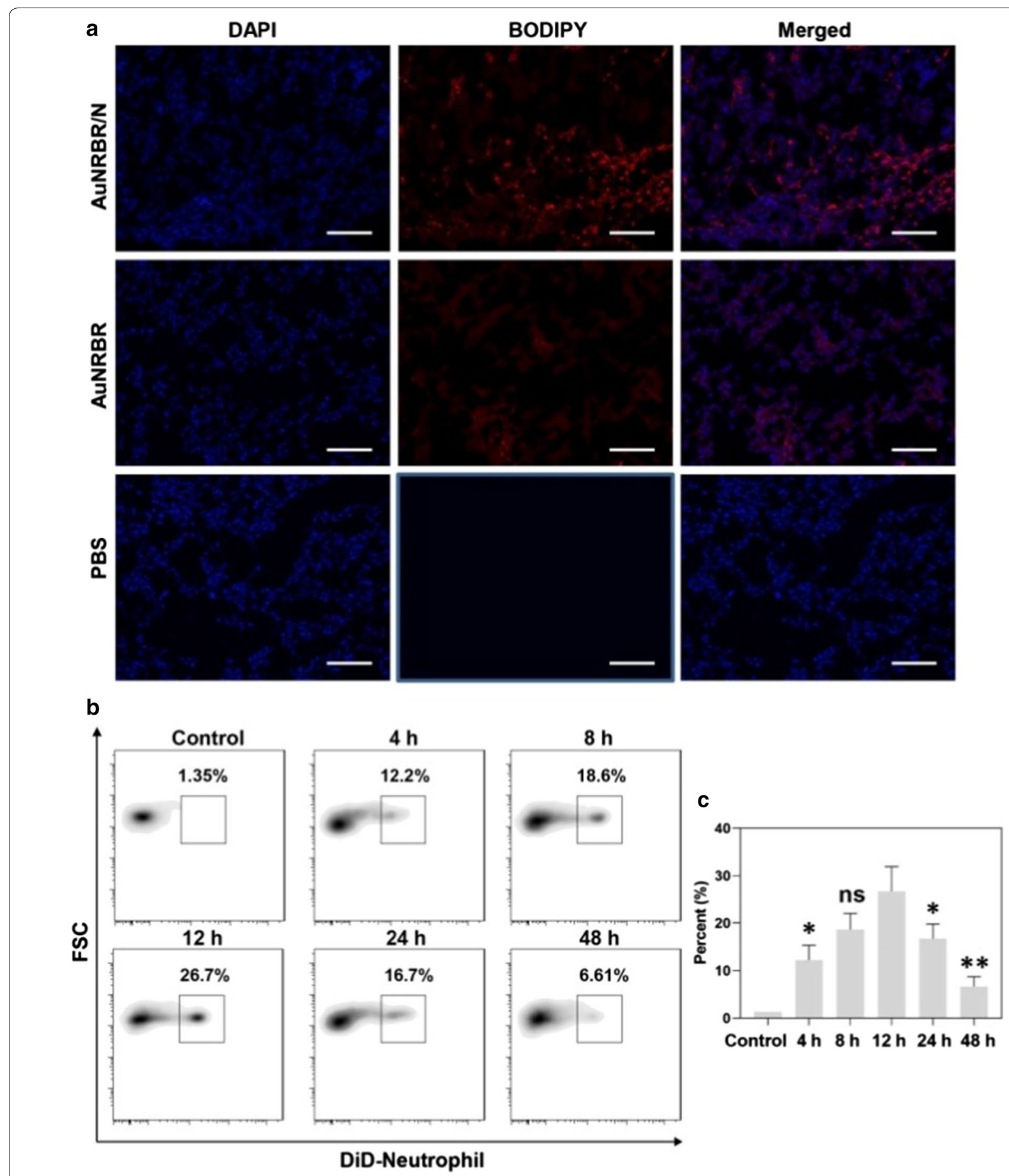

Fig. 6 a CLSM images of tumor tissues in the Lewis tumor-bearing mice after injection of AuNRBR/N, AuNRBR and PBS. Blue fluorescence: nuclei, red fluorescence: BODIPY. The ratio of administered neutrophils in the total neutrophils in tumor after intravenous injection of AuNRBR/N labeled with DiD at 4, 8, 12, 24 and $48 \mathrm{~h}$. The percent showed by $\mathbf{b}$ representative flow cytometry data and individual histograms. Scale bar: $100 \mu m^{*} p<0.05$ and ${ }^{* *} p<0.01$ vs the 12 h group 
a

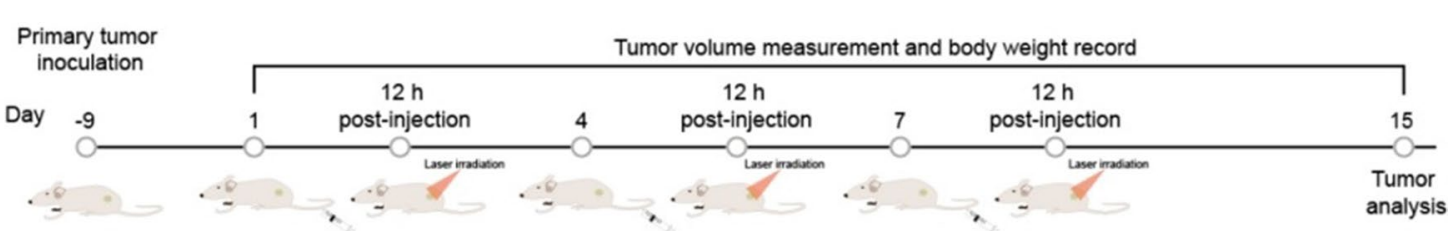

b

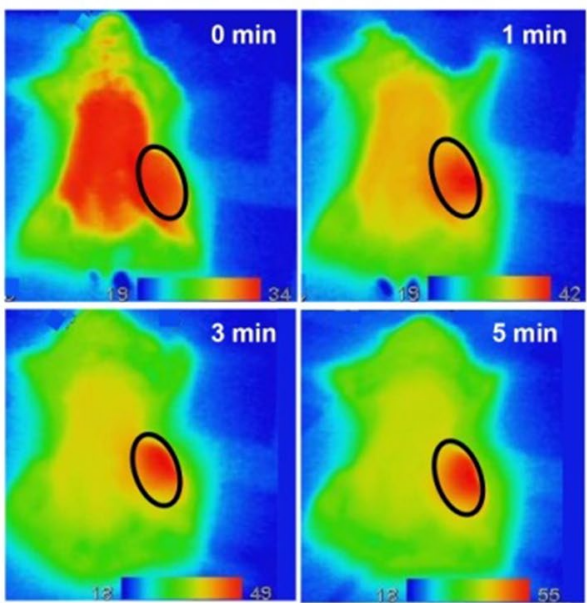

C

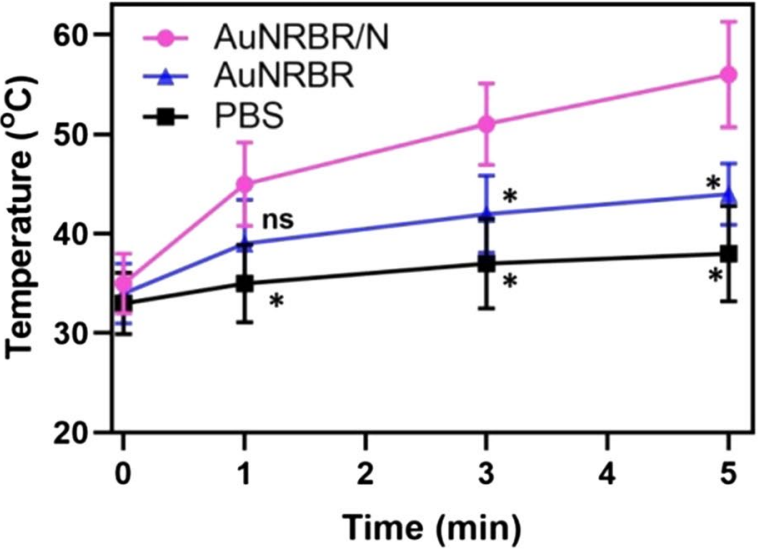

Fig. 7 a Schematic diagram of the experimental design. $\mathbf{b} \mid \mathrm{R}$ thermal images and $\mathbf{c}$ temperature changing curve of Lewis tumor bearing mice treated with AuNRBR/N under $808 \mathrm{~nm}$ laser irradiation within $5 \mathrm{~min}\left(0.5 \mathrm{~W} / \mathrm{cm}^{2}\right) .{ }^{*} \mathrm{p}<0.05$ vs the AuNRBR/N group

in the tumour originated from AuNRBR/N at $12 \mathrm{~h}$ postinjection. The ratio of exogenously injected neutrophils decreased after $12 \mathrm{~h}$ and descended to around $5 \%$ at $48 \mathrm{~h}$ post-injection (Fig. 6c). The AuNRBR/N could be effectively recruited into the tumour and eliminated gradually.

\section{AuNRBR/N induce potent antitumour therapy in vivo}

AuNR based PTT drug delivery system can induce apoptosis or necrosis of the cancer cells and suppress tumor growth by generating a localized hyperthermia effect. However, the lack of specificity of the AuNR would lead to inevitable damage to surrounding healthy tissues. The antitumour therapy experiment was designed as a schematic diagram shown in Fig. 7a. Firstly, the temperature of the Lewis tumour was detected with laser irradiation after administration of AuNRBR or AuNRBR/N. As shown in Fig. $7 \mathrm{~b}$, the temperature of the Lewis tumour increased up to $55^{\circ} \mathrm{C}$ within $5 \mathrm{~min}$ when exposed to the $808 \mathrm{~nm}$ laser irradiation $\left(0.5 \mathrm{~W} / \mathrm{cm}^{2}\right)$, thereby exhibiting a stronger photothermal therapeutic efficiency of AuNRBR/N to the tumour. Interestingly, AuNRBR/N showed a more significantly enhanced photothermal effect than AuNRBR, which induced the temperature increase to around $42{ }^{\circ} \mathrm{C}$ within $5 \mathrm{~min}$ (Fig. 7c). The elevated temperature confirmed the PTT efficiency of AuNRBR/N.
Then, the sizes of tumours were continuously monitored for 15 days. As shown in Fig. 8a, b, the tumour volume of mice injected with PBS quickly increased, which indicated that laser irradiation alone had no therapeutic effect on the tumour. However, the tumour of AuNRtreated mice was inhibited modestly compared with PTT alone due to limited EPR effect. Moreover, the tumour growth was obviously delayed when the mice were administrated with AuNRBR, thereby suggesting the active targeting ability of AuNRBR. Apparently, complete suppression of the growth of tumour treated with AuNR or AuNRBR was difficult, thereby indicating the limited targetability, which contributed to the undesired therapeutic effect. However, the tumour growth of mice was significantly inhibited when injected with AuNRBR/N. The enhanced therapeutic effect for AuNRBR/N was ascribed to the remarkable targeting efficiency of neutrophil, thereby leading to a higher amount of AuNRBR/N recruited into the tumour. AuNRBR/N would further release AuNRBR for penetration into deep tumour tissue, thereby leading to a robust therapeutic effect. The body weight was also stable during the therapy, which suggested the biosafety of AuNRBR/N (Fig. 8c). As seen in Fig. $8 \mathrm{~d}$, the median survival increased from 55 days for the control group to 60 days for the AuNR group. A more significant increase in the median survival (95 days) was observed in the AuNRBR group. For the 

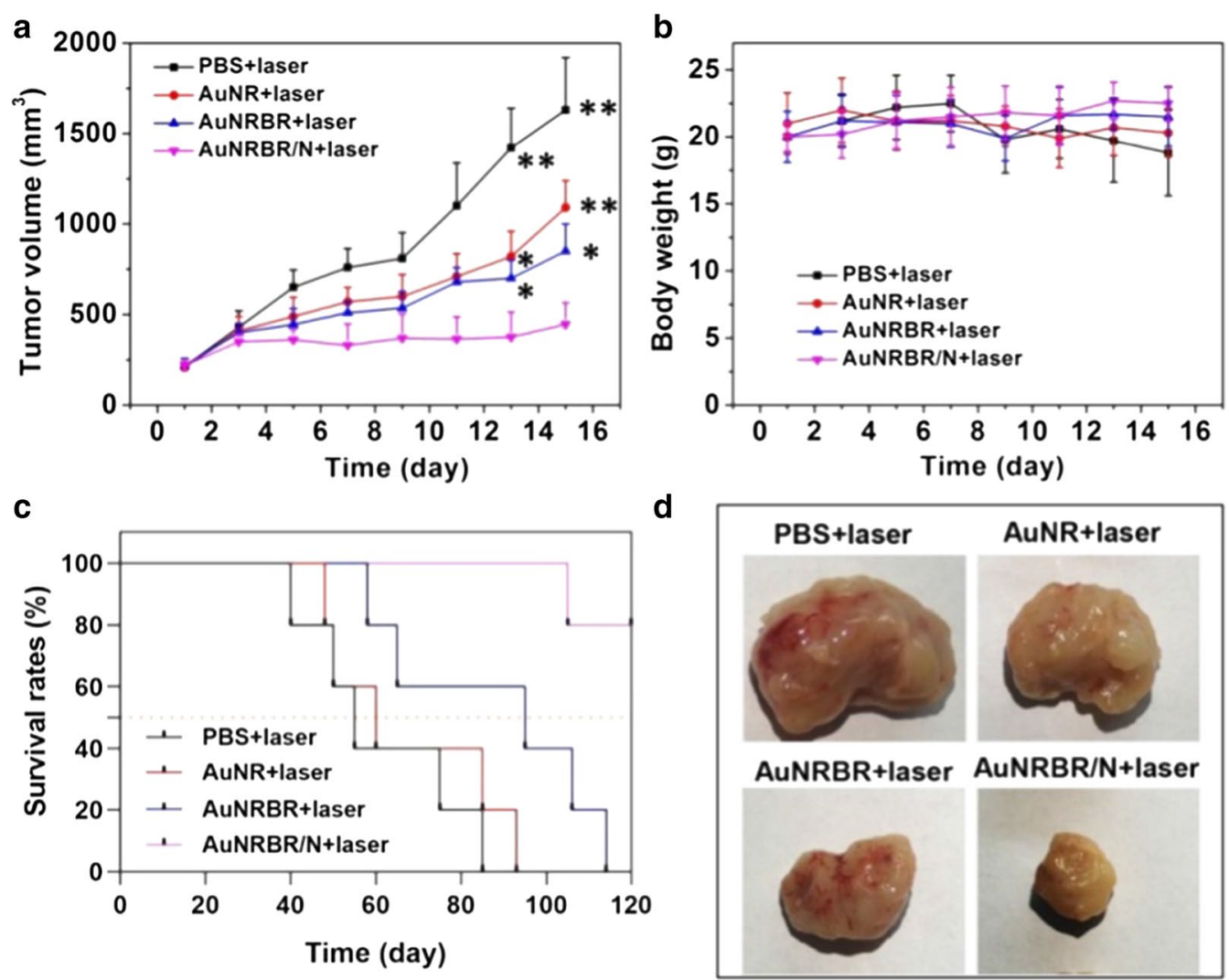

Fig. 8 a Tumor growth curve and $\mathbf{b}$ body weight change of mice with different treatments during 15 days. $\mathbf{c}$ Survival curves of Lewis tumor-bearing mice after various treatments. AuNRBR/N injected mice showed improved survival over 120 days after treatments. $\mathbf{d}$ The images of tumor in mice on day 15 after various treatments. Scale bar: $100 \mu \mathrm{m}$. The error bars were presented as mean $\pm \operatorname{SD}(n=5)$. ${ }^{*} p<0.05$ and ${ }^{* *} p<0.01 \mathrm{vs}$ the AuNRBR/N + laser group

AuNRBR/N-treated group, the median survival reached over 120 days. The administration of AuNRBR/N led to a significant increase in median survival of Lewis tumourbearing mice. Thus, AuNRBR/N can significantly inhibit the growth of the tumour due to the neutrophil-based multistage delivery system and the improved active targeting of AuNRBR to the tumour. To study the potential toxicity, major organs (heart, liver, spleen, lung and kidney) were stained with hematoxylin and eosin (H\&E). As shown in Fig. 9, no significant microscopic lesions were revealed in all three groups during 15 days compared with the control group, indicating that AuNRBR/N was biocompatible and safe.

\section{Conclusions}

We developed a novel cell-based multistage nanoparticle delivery system for PTT of cancer. The AuNR was successfully synthesised and modified with BSA. AuNRB modified with RGD can increase the targeting efficiency and evoke the intensive antitumour capability in vitro, which may increase penetration in tumour site after neutrophil delivering AuNRBR. Additionally, AuNRBR/N could migrate across the epithelial cells efficiently, had better toxicity against Lewis cells in vitro and enhanced tumour targeting efficiency in vivo. More importantly, AuNRBR/N exhibited superior therapeutic effect on 


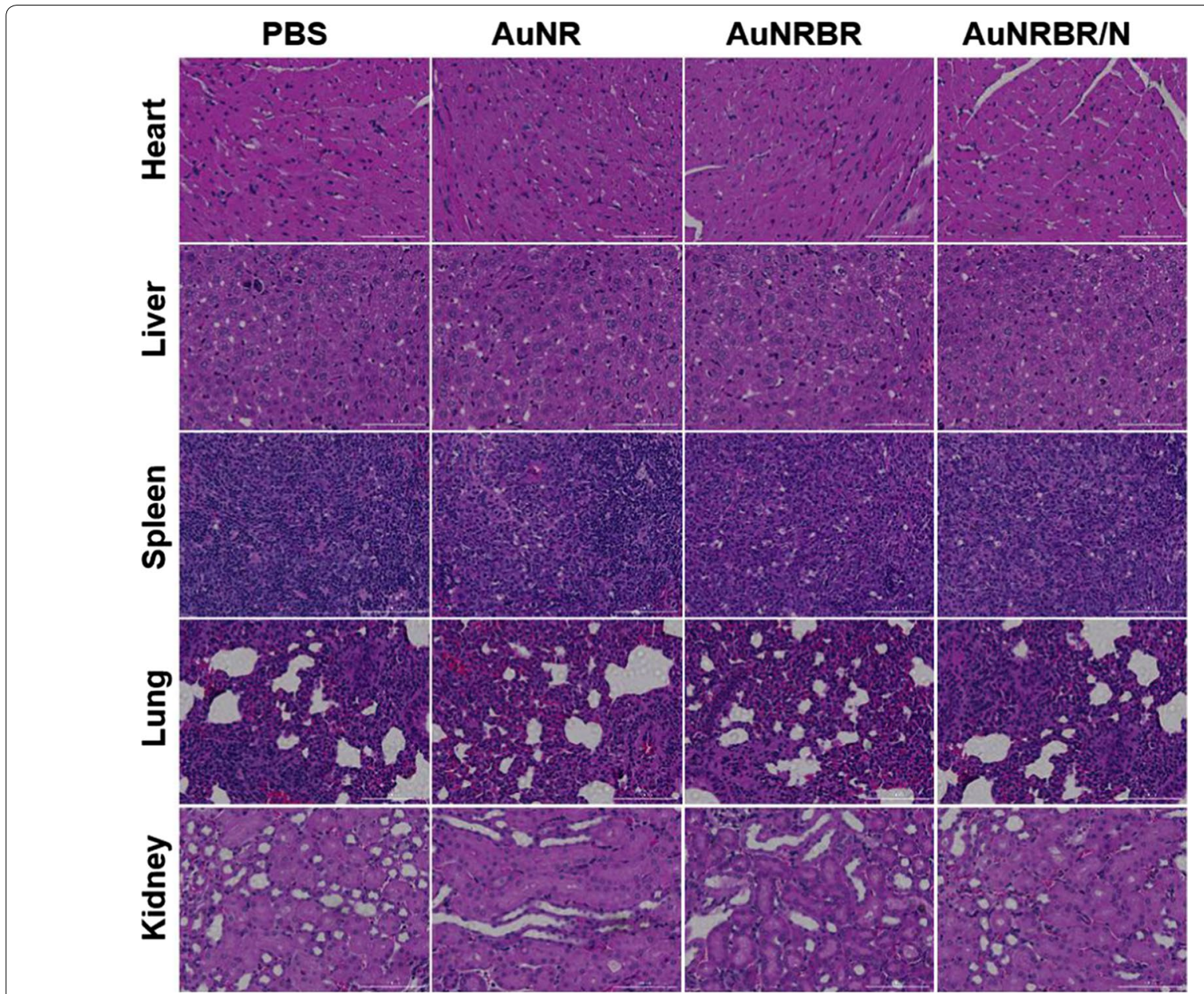

Fig. 9 The HE staining of main organ sections at day 15 after administration of different treatments. Scale bar: $100 \mu m$

Lewis tumour due to the optimal tumour targeting of neutrophils and multistage delivery of AuNRBR for deep tumour diffusion, which also improved the survival rate of mice (over 120 days). We hope this novel cell-based multistage AuNR delivery system can provide a new strategy for cancer PTT.

\section{Abbreviations}

PTT: Photothermal therapy; AuNR: Au nanorod; NIR: Near-infrared; MPS: Mononuclear phagocytic system; FBS: Foetal bovine serum; DAPI: 4,6-diamidino-2-phenylindole; BSA: Bovine serum albumin; CTAB: Hexadecyl trimethyl ammonium bromide; DMSO: Dimethyl sulfoxide; FITC: Fluorescein5(6)isothiocyanate; CLSM: Confocal laser scanning microscopy; HUVECs: Human umbilical vein endothelial cells.

\section{Acknowledgements}

This work was supported by the Program in the Fundamental Research Funds for the Central Universities (1501219171), Natural Science Foundation of China $(81572245,81402571,81903172,81903142)$, Western Medicine approved by Shanghai Science and Technology Commission (16411966000), the Interdisciplinary Program of Shanghai Jiaotong University (YG2014QN22),Science and Technology Comission Shanghai Municipality(STCSM15DZ2270400), Collaborative Research Project of Transformational Medicine Collaborative Innovation Center (TM201822) and the Natural Science Foundation of Anhui Province [KJ2019A0293].

\section{Authors' contributions}

$\mathrm{BY}, \mathrm{BZ}$ and FW contributed equally to this project. AL, LZ and JQ performed the conception and design of the study. BY, BZ, FW, KW, YG and QL performed materials preparation, in vitro and in vivo experiments, and statistical analysis. $B Z, F W$ and $K W$ performed image analyses. $A L, L Z, J Q$, and $B Y$ wrote the manuscript. AL, LZ and JQ supervised the entire study, provided the financial support. All authors read and approved the final manuscript.

\section{Ethics approval and consent to participate}

Not applicable.

\section{Competing interests}

The authors declare no competing financial interest.

\section{Author details}

${ }^{1}$ Department of Thoracic Surgery, Shanghai Chest Hospital, Shanghai Jiao Tong University, Shanghai, China. ${ }^{2}$ Department of Otorhinolaryngology Head and Neck Surgery, First Affiliated Hospital of Bengbu Medical College, Bengbu, China. ${ }^{3}$ Cancer Center, Shanghai East Hospital, Tongji University, Shanghai, China. ${ }^{4}$ Department of Thoracic Surgery, Pizhou people's hospital, Xuzhou Medical University, Pizhou, China. ${ }^{5}$ Shanghai Tenth People's Hospital, School of Medicine, Tongji University, Shanghai 200072, People's Republic of China. ${ }^{6}$ School of Life Science and Technology, Tongji University, 1239 Siping Road, Shanghai 200092, People's Republic of China. ${ }^{7}$ Department of Respiratory Medicine, Shanghai Ninth Peoples Hospital, Shanghai Jiao Tong University School of Medicine, 639 Zhizaoju Rd, Shanghai 200011, China. 
Received: 26 May 2020 Accepted: 25 August 2020

Published online: 29 September 2020

\section{References}

1. Song J, Yang X, Jacobson O, Huang P, Sun X, Lin L, Yan X, Niu G, Ma Q, Chen X. Ultrasmall Gold Nanorod Vesicles with Enhanced Tumor Accumulation and Fast Excretion from the Body for Cancer Therapy. Adv Mater. 2015;27:4910-7.

2. Gustafson $\mathrm{HH}$, Holt-Casper D, Grainger DW, Ghandehari $\mathrm{H}$. Nanoparticle uptake: The phagocyte problem. Nano Today. 2015;10:487-510.

3. Bourzac K. Cancer nanomedicine, reengineered. Proc Natl Acad Sci USA. 2016;113:12600-3.

4. Kooijmans SA, Fliervoet LA, van der Meel R, Fens MH, Heijnen HF, van Bergen En Henegouwen PM, Vader P, Schiffelers RM. PEGylated and targeted extracellular vesicles display enhanced cell specificity and circulation time. J Control Release. 2016:224:77-85.

5. Patil Y, Amitay Y, Ohana P, Shmeeda H, Gabizon A. Targeting of pegylated liposomal mitomycin-C prodrug to the folate receptor of cancer cells: intracellular activation and enhanced cytotoxicity. J Control Release. 2016:225:87-95.

6. Lee JY, Termsarasab U, Park JH, Lee SY, Ko SH, Shim JS, Chung SJ, Cho HJ, Kim DD. Dual CD44 and folate receptor-targeted nanoparticles for cancer diagnosis and anticancer drug delivery. J Control Release. 2016;236:38-46.

7. Srinivasarao M, Low PS. Ligand-targeted drug delivery. Chem Rev. 2017;117:12133-64.

8. Mangadlao JD, Wang X, McCleese C, Escamilla M, Ramamurthy G, Wang Z, Govande M, Basilion JP, Burda C. Prostate-specific membrane antigen targeted gold nanoparticles for theranostics of prostate cancer. ACS Nano. 2018;12:3714-25.

9. Sun Q, Zhou Z, Qiu N, Shen Y. Rational design of cancer nanomedicine: nanoproperty integration and synchronization. Adv Mater. 2017;29:1606628.

10. Huang WC, Chiang WH, Cheng YH, Lin WC, Yu CF, Yen CY, Yeh CK, Chern CS, Chiang CS, Chiu HC. Tumortropic monocyte-mediated delivery of echogenic polymer bubbles and therapeutic vesicles for chemotherapy of tumor hypoxia. Biomaterials. 2015;71:71-83.

11. Choi J, Kim H-Y, Ju EJ, Jung J, Park J, Chung H-K, Lee JS, Lee JS, Park HJ, Song SY. Use of macrophages to deliver therapeutic and imaging contrast agents to tumors. Biomaterials. 2012;33:4195-203.
12. Huang B, Abraham WD, Zheng YR, Lopez SCB, Luo SS, Irvine DJ. Active targeting of chemotherapy to disseminated tumors using nanoparticlecarrying T cells. Sci Transl Med. 2015;7:291 ra94.

13. Zhao Z, Zhang L, Xue L, Shen S, Wen Y, Wei Z, Wang L, Kong L, Sun H, Ping Q, Mo R, Zhang C. Neutrophil-mediated anticancer drug delivery for suppression of postoperative malignant glioma recurrence. Nat Nanotech. 2017;12:692-700.

14. Stephan MT, Moon JJ, Um SH, Bershteyn A, Irvine DJ. Therapeutic cell engineering with surface-conjugated synthetic nanoparticles. Nat Med. 2010;16:1035-41.

15. Poon IKH, Lucas CD, Rossi AG, Ravichandran KS. Apoptotic cell clearance: basic biology and therapeutic potential. Nat Rev Immunol. 2014;14:166-80.

16. Timin AS, Litvak MM, Gorin DA, Atochina-Vasserman EN, Atochin DN, Sukhorukov GB. Cell-based drug delivery and use of nano- and microcarriers for cell functionalization. Adv Healthc Mater. 2018;7:1700818.

17. Wu M, Zhang H, Tie C, Yan C, Deng Z, Wan Q, Liu X, Yan F, Zheng H. MR imaging tracking of inflammation-activatable engineered neutrophils for targeted therapy of surgically treated glioma. Nat Commun. 2018:9:4777.

18. Wang FH, Huang Q, Wang Y, Shi LL, Shen YY, Guo SR. NIR-light and GSH activated cytosolic p65-shRNA delivery for precise treatment of metastatic cancer. J Control Release. 2018;288:126-35.

19. Brinkmann V, Reichard U, Goosmann C, Fauler B, Uhlemann Y, Weiss DS, Weinrauch Y, Zychlinsky A. Neutrophil extracellular traps kill bacteria. Science. 2004;303:1532-5.

20. Nikoobakht B, El-Sayed MA. preparation and growth mechanism of gold nanorods (NRs) using seed-mediated growth method. Chem Mater. 2003:15:1957-62.

21. Ali MRK, Panikkanvalappil SR, El-Sayed MA. Enhancing the efficiency of gold nanoparticles treatment of cancer by increasing their rate of endocytosis and cell accumulation using rifampicin. J Am Chem Soc. 2014;136(12):4464-7.

\section{Publisher's Note}

Springer Nature remains neutral with regard to jurisdictional claims in published maps and institutional affiliations.

Ready to submit your research? Choose BMC and benefit from

- fast, convenient online submission

- thorough peer review by experienced researchers in your field

- rapid publication on acceptance

- support for research data, including large and complex data types

- gold Open Access which fosters wider collaboration and increased citations

- maximum visibility for your research: over 100M website views per year

At BMC, research is always in progress.

Learn more biomedcentral.com/submissions 